\title{
Will the use of a raised or age-adjusted D-dimer cut-off value in combination with a sequential strategy using the Wells score safely exclude deep venous thrombosis?
}

\author{
Ramberg E ${ }^{1}$, Engell AE ${ }^{2}$, Osterskov MW', Sundskaro MM'1 and Bathum L ${ }^{2}$ \\ ${ }^{1}$ Department of Medicine, Amager and Hvidovre Hospital, Denmark \\ ${ }^{2}$ Department of Clinical Biochemistry, Amager and Hvidovre Hospital, Denmark
}

\begin{abstract}
Introduction: The reference interval for D-dimer is increasing with age, making a fixed cut-off value for venous thromboembolism (VTE) less appropriate, as the number of VTE increases with age. We aimed to test whether a raised (0.9 FEU/L) or age-related (0.01*age FEU/L in age $>50$ years) D-dimer cut-off limit safely excludes deep venous thrombosis (DVT) after a risk estimation using Wells score in a population admitted to an emergency department with a suspicion of (DVT). D-dimer should be used to rule out DVT in groups with intermediate or low pre-test probability of DVT using the Wells score.
\end{abstract}

Method: We retrospectively validated 3,499 patients admitted to two separately emergency departments from July 1, 2013 to December 31, 2015 for possible DVT. The patient records were evaluated in all patients with a D-dimer $\geq 0.5 \mathrm{FEU} / \mathrm{L}$, and data were obtained regarding Wells scores and ultrasonography (US). D-dimers were measured by HemosIL D-dimer.

Result: Slightly more women were included; however, slightly more men had a positive US. In the total population; 6 out of 42 (14\%) with a D-dimer between 0.5 and $0.9 \mathrm{FEU} / \mathrm{L}$ had an US indicative of DVT in the group with low Wells score and 19 out of 62 (31\%) in the group with intermediate Wells score. For the age-related cut-off, the figures were 3 of 20 (15\%) in the group with low Wells score and 11 out of 36 (31\%) in the group with intermediate Wells score.

Conclusion: This retrospective study does not support that a higher cut-off level for D-dimer in the groups with low or intermediate Wells scores, are safe to exclude the diagnosis of DVT in an emergency department.

\begin{abstract}
Abbreviations: VTE-Venous Thromboembolism; DVT-Deep Vein Thrombosis; FEU-Fibrinogen Equivalent Unit; US- Ultrasonography; PE- pulmonary embolism.
\end{abstract}

\section{Background}

The diagnosis of deep vein thrombosis (DVT) is based on a sequential strategy involving a clinical pre-test probability assessment with the use of Wells scores [1,2], D-dimer testing, and if necessary, a compression ultrasonography (US) $[3,4]$. D-dimer is a rather unspecific fibrin related degradation marker, being elevated in many clinical situations (e.g., infection, inflammation, malignancy, postsurgical status, and pregnancy) and whenever there is acute thrombus in the vasculature [4]. Many D-dimer tests are available; however, external quality assessment programmes in Europe have shown poor comparability of results between methods, reflecting poor standardization of these tests $[5,6]$. The specificity of the D-dimer test to rule out DVT have been shown to be strongly dependent on the pre-test clinical probability of DVT [7]. Thus D-dimer should optimally be used to rule out DVT in the group with intermediate or low pre-test probability of DVT using the Wells score $[1,8,9]$.

The most commonly used cut-off for the exclusion of venous thromboembolism (VTE) is a D-dimer value of $0.5 \mathrm{FEU} / \mathrm{L}$ [10]. Since $\mathrm{D}$-dimer increases with age $[11,12]$, D-dimer is less useful for excluding VTE in the elderly. Furthermore, the incidence of VTE increases markedly by age, with an increase in the hazard ratio of 1.69 per 10 -year increase in age [13]. These two facts question the suitability of the cut- off at $0.5 \mathrm{FEU} / \mathrm{L}$ in an ageing population. To increase the effectiveness of D-dimer to exclude the diagnosis of VTE in older patients, several studies have suggested age-dependent cut-off limits [14]. Harper et al. proposed a cut-off value of $500 \mu \mathrm{g} / \mathrm{L}$ or $0.5 \mathrm{FEU} / \mathrm{L}$ in patients $<60$ years and $1,000 \mu \mathrm{g} / \mathrm{L}$ or $1.0 \mathrm{FEU} / \mathrm{L}$ for the elderly [11]. Haas et al. [15] suggested a firm cut-off limit of $750 \mu \mathrm{g} / \mathrm{L}$ or $0.75 \mathrm{FEU} / \mathrm{L}$ in patients $60+$ years. Years and Raviv [16] suggested that the cut-off limit should be increased to $900 \mu \mathrm{g} / \mathrm{L}$ or $0.9 \mathrm{FEU} / \mathrm{L}$ in all age groups. Douma et al. proposed a dynamic limit (patient's age $\left.{ }^{\star} 10\right) \mu \mathrm{g} / \mathrm{L}$ or (patient's age ${ }^{\star}$ $0.01) \mathrm{FEU} / \mathrm{L}$ at $50+$ year $[17,18]$.

Several studies recommend the use of the age-adjusted D-dimer cutoff in the diagnostic strategy for DVT and find that the dynamic limit suggested by Douma (patient's age * 0.01) FEU/L is just as safe as the standard D-dimer cut-off value to rule out DVT in the elderly $[19,20]$. In this study, we aimed to test whether a raised $(0.9 \mathrm{FEU} / \mathrm{L})$ limit in all age groups or the age-related D-dimer ((patient's age * 0.01) FEU/L at $50+$ year) cut-off limit in combination with the clinical probability test (Wells score); safely exclude DVT in a population admitted to the hospital with a suspicion of DVT.

${ }^{\star}$ Correspondence to: Emilie Ramberg, Kettegaard alle, Hvidovre, Denmark, E-mail: eram0001@regionh.dk

Key words: D-dimer, venous thrombosis, ultrasonography, wells score

Received: July 11, 2018; Accepted: July 20, 2018; Published: July 23, 2018 


\section{Material and method}

\section{Patient population and data collection}

We retrospectively evaluated all patients above 18 years of age for possible DVT who were referred to the Emergency Department at Amager or Hvidovre Hospital between January 1, 2013 and December 31,2015 . Both hospitals are situated in the Capital Region of Denmark. Hvidovre hospital has more than 60,000 admissions per year and Amager Hospital has approximately 30,000 admissions per year. Together, they provide healthcare sevices for approximately 500,000 citizens.

All admission diagnoses are classified according to the Danish version of the International Classification of Diseases, Tenth revision (ICD10). In this study, we included all patients with one of the following diagnosis: pain in limb, hand, foot, fingers and toes (DM 796), phlebitis and thrombophlebitis of other and unspecified deep vessels of lower extremities (DI 802), phlebitis and thrombophlebitis of lower extremities, unspecified (DI 803), phlebitis and thrombophlebitis of other sites (DI 808), and phlebitis and thrombophlebitis of superficial vessels of lower extremities (DI 800). Recorded information includes the 10-digit Civil Personal Registration number (CPR) unique to every Danish resident as well as the dates of admission and discharge. The CPR number was used to link information on all patients older than 18 years between the different registries. Data regarding D-dimers were drawn from the Laboratory Information Management System (LABKA) that is common in these two hospitals. The D-dimer had to be measured within 24 hours after admission. Only the first admission was included in patients who had several admissions and only patients with a suspected DVT in the lower limbs were included. The patient records were evaluated in all patients with a D-dimer $\geq 0.5 \mathrm{FEU} / \mathrm{L}$, and data were obtained regarding Wells score and ultrasonography (US). Only the first ultrasonography was recorded. Pregnant women were excluded from our analysis.

\section{Ultrasonography}

The ultra-sonographic duplex lower-limb compression test was performed with the Philips XD11XE system using a linear transducer according to the standard protocol which was a whole leg ultrasound. A positive test indicated a diagnosis of DVT. Inconclusive tests were referred to as positive. A distinction between proximal and distal DVT was not made.

\section{D-dimer measurement}

D-dimers were measured by HemosILD-dimer HS (Instrumentation Laboratory, U.S.A.) on an ACL Top 500 (Instrumentation Laboratory, U.S.A.) in both laboratories. The intermediary imprecision was $4.6 \%$ at $0.71 \mathrm{FEU} / \mathrm{L}$ and $3.9 \%$ at $1.5 \mathrm{FEU} / \mathrm{L}$ during the testing period.

\section{Wells score}

We used the simplified Wells score to categorize patients with a low, intermediate or high DVT probability [4]. The Wells score documented in the patient record was noted. In patients for whom the Wells score was not documented, the score was retrospectively calculated using data available from the admission notes $[1,21]$.

\section{Statistics}

The difference in the proportion of positive US in the two sexes was tested by the two-sample test of proportion. StataSE 13 was used for the statistical calculations and the graphical presentation.

\section{Ethics}

The study was approved by the Danish Data Protection Agency (number: 2012-58-0004; AHH-2015-094; I-Suite number: 04194). Furthermore The Danish Health authority gave permission to evaluate the records of the included patient's (j.number: 3-3013-1362/1). According to Danish law, epidemiological studies do not need approval by an Ethics Committee.

\section{Result}

The descriptive data of the study is shown in Table 1. In total, 9 subjects ( 7 males and 2 females) did not have a Wells score, and data in the records were not sufficient to determine a score. It is recognizable that more females than males were referred to the emergency departments with the chosen diagnoses. Females tended to be a little older at referral. However, the distribution changes as an equal number of males and females had a D-dimer measurement and an US. The sex distribution among positive US deviated from the expected, with a significant higher proportion of positive US among males $(\mathrm{p}<0.001)$.

Table 2 shows the distribution of a positive and a negative US divided in Wells score low, intermediate and in a combination of the two scores. According to the sequential strategy, almost all subjects in our study with a Wells score of low or intermediate risk and a D-dimer $\geq 0.5 \mathrm{FEU} / \mathrm{L}$ had a diagnostic US performed. The percentage of false negatives (D-dimer below the cut-off but a positive US) are given in Table 2. There seem to be more false negatives in the group with an intermediate Wells score compared to a low Wells score. However, the percentages of false negatives were quite high - between 14 and $31 \%$. Figure 1 shows the distribution graphically in the combined Wells scores low and intermediate.

Table 1. Descriptive data from the total population

\begin{tabular}{|l|c|c|c|}
\hline & $\begin{array}{c}\text { Males } \\
(\mathbf{N}=\mathbf{1 , 4 8 5})\end{array}$ & $\begin{array}{c}\text { Females } \\
(\mathbf{N = 2 , 0 1 4 )}\end{array}$ & $\begin{array}{c}\text { Total } \\
(\mathbf{N}=\mathbf{3 , 4 9 9})\end{array}$ \\
\hline Total population & $52.6(53)$ & $55.3(56)$ & $54.2(54)$ \\
\hline Age mean (median) & $18-98$ & $18-103$ & $18-103$ \\
\hline Age range & $446(30.0)$ & $563(28.0)$ & $1,009(28.8)$ \\
\hline Individuals with a D-dimer measurement & $59(58.5)$ & $59(59.2)$ & $59(58.9)$ \\
\hline Numbers (\% of total) & $19-98$ & $20-101$ & $19-101$ \\
\hline Age (median) & $256(17.2)$ & $252(12.5)$ & $508(14.5)$ \\
\hline Age range & $60.0(60)$ & $65.0(71)$ & $62.5(65)$ \\
\hline Individuals with a D-dimer measurement and an ultrasonography & $19-98$ & $20-101$ & $19-101$ \\
\hline Numbers (\% of total) & $168(11.3)$ & $115(5.7)$ & $283(8.1)$ \\
\hline Age (median) & $54(21.7)$ & $56(22.4)$ & $110(22.0)$ \\
\hline Age range & $113(45.4)$ & $135(54.0)$ & $248(49.7)$ \\
\hline Positive US; N (\% of total) & $82(32.9)$ & $59(23.6)$ & $141(28.3)$ \\
\hline Wells score low; N (\% of Wells) &
\end{tabular}

Table 2. Distribution of positive and negative ultrasonographies in the area between the current cut-off at $0.5 \mathrm{FEU} / \mathrm{L}$ and the two different cut-offs $(0.9 \mathrm{FEU} / \mathrm{L}$ and the age-related cut-off) in Wells score low, intermediate (IM) and the sum of the two groups

\begin{tabular}{|c|c|c|c|c|c|c|}
\hline Cut-off & \multicolumn{3}{|c|}{$0.5 \geq \&<0.9 \mathrm{FEU} / \mathrm{L}$} & \multicolumn{3}{|c|}{ Age related cut-off' ${ }^{1}$} \\
\hline Wells score & Low & IM & Low+IM & Low & IM & Low+IM \\
\hline Total US (N) & 42 & 62 & 104 & 20 & 36 & 56 \\
\hline Positive US ${ }^{2}(\%)$ & $6(14 \%)$ & $19(31 \%)$ & $25(24 \%)$ & $3(15 \%)$ & $11(31 \%)$ & $14(25 \%)$ \\
\hline Negative US & 36 & 43 & 79 & 17 & 25 & 42 \\
\hline
\end{tabular}

${ }^{1}$ Age-related cut-off: $0.01^{*}$ age is only calculated if age is above 50 .

${ }^{2} \mathrm{An}$ ultrasonography described as inconclusive was considered positive. The numbers are the positive between $0.5 \mathrm{FEU} / \mathrm{L}$ and the cut-off. 


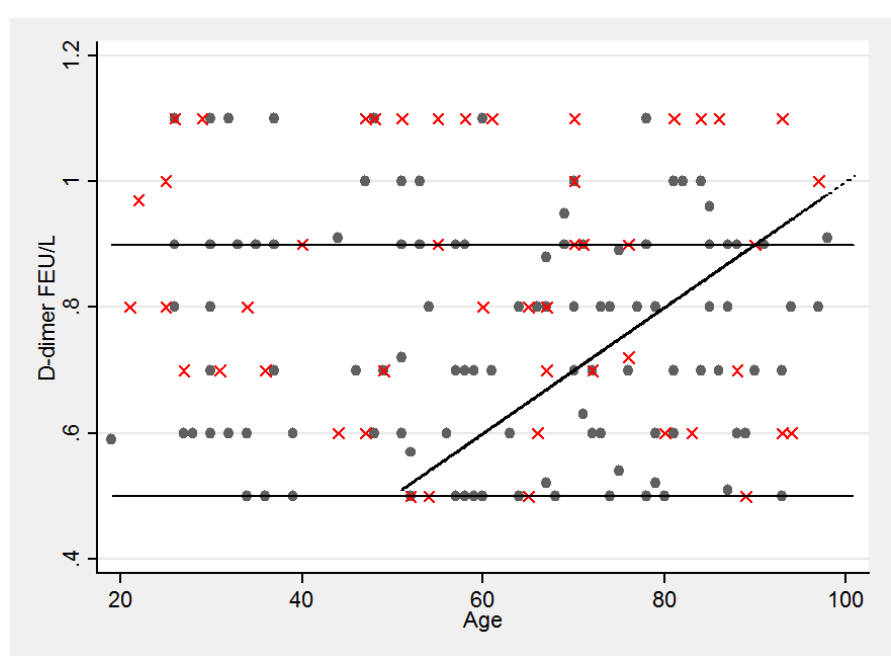

Figure 1. Distribution of D-dimers in the group with ultrasonography and Wells scores low and intermediate

D-dimer values are truncated at $2 \mathrm{FEU} / \mathrm{L}$. The 3 different cut-off levels are shown. Dots present negative and $\mathrm{X}^{\prime}$ s positive ultrasonography

\section{Discussion}

This study investigated whether the widely used D-dimer cut-off value at $0.5 \mathrm{FEU} / \mathrm{L}$ could be raised in all age groups or alternatively increased as a function of age and still safely excludes DVT in the elderly. Slightly more women were included in our study; however, slightly more men had a positive US. Women were marginally older compared to males. This difference in age distributions between the genders could partly explain the higher admission rate among women but does not explain the apparently higher proportion of DVT among those with an US in males. However, it is known that sex-specific differences for VTE exist, with a higher risk in women during their fertile life [22] and a higher risk in men at older ages [23]. Thus, our findings are in accordance with previous studies. The sex distribution in the admitted population was probably affected by the fact that the selected inclusion diagnoses were not specific for DVT, making the difference less relevant.

Previous studies have shown that reference intervals for D-dimers are age-dependent with a marked increase by age [12], and establishing a fixed cut-off value is less appropriate, as the amount of DVT increases with age [24]. It has been shown that the number of healthy individuals with a D-dimer above $0.5 \mathrm{FEU} / \mathrm{L}$ easily can exceed $50 \%$ in a population above 70 years [12]. An increase in the D-dimer cut-off level for older patients suspected of a DVT could lead to a reduction in admission rates, use of antithrombotic medicine and radiological investigations. Since pulmonary embolism (PE), in most cases, is the consequence of a DVT, it is essential to identify subjects with a DVT [9]. However, our study showed that the two examined cut-off values falsely reject DVT in a fairly high percentage of individuals, even in the low risk group of patients defined by the Wells score.

Finding that the age-adjusted cut-off suggested by Douma $[17,18]$, does have a proportion of false positives up to $31 \%$ is quite surprising, as a previous study showed that the age-adjusted D-dimer substantially increases the number of patients above 50 years in whom PE can be safely excluded [25]. This has also been revealed to be the case in cancer patients $[26,27]$. Furthermore, one study indicated that the cut-off level could be raised to age ${ }^{\star} 0.025 \mathrm{FEU} / \mathrm{L}\left(\operatorname{age}^{\star} 25 \mu \mathrm{g} / \mathrm{L}\right)$ in the lowest probability group (Wells low risk score) without jeopardizing safety in diagnosing DVT [28]. One study compared five different D-dimer reagents and the application of an age-adjusted cut-off for the diagnosis of VTE in emergency departments and found that the age-adjusted method was cost-effective without significantly increasing the number of false negatives [6]. This was also perceived for HemosIL D-dimers, which was the test used in our study. The conflicting results emphasizes that care should be taken before changing D-dimer thresholds. Every hospital has to evaluate the possible consequences before implementing the change. There is a poor comparability between the different $\mathrm{D}$-dimer assays [5,6], and care should be taken in the case of a change in D-dimer analysis.

One previous study compared three different D-dimer cut-off strategies in diagnosing VTE; one with a general increase in cut-off, the age-adjusted cut-off suggested by Douma also investigated in this study and a reverse age-adjusted cut-off [29]. They found no support for the use of a progressively higher D-dimer level to exclude DVT with increasing age; however, their study suggested that the D-dimer threshold could be generally increased in all age groups without negative consequences. This is contrary to our findings that an increase in cutoff provides more false negatives. One reason for this discrepancy could be that the study by Lapner was restricted to outpatients and had a fairly low frequency of DVT at $4.2 \%$, however the present study focused on admitted patients with a frequency of positive US at $8.1 \%$ at the first radiologic examination [29].

The pre-test clinical probability measurement with the Wells score has been widely investigated and found to be helpful in the diagnosis of proximal deep vein thrombosis of the lower limb [1]. Wells scores were proven to have a fairly high interobserver agreement [30]. However, studies have questioned whether Wells is the best measurement to make a pre-test clinical estimation, and they revealed that empirical clinical estimation based on the clinician's experience could be superior [30]. Furthermore, the value of Wells scores seemed to be low in isolated distal DVT [31]. Our study included both proximal and distal DVT, which could make the Wells scores less useful.

The present work has several limitations. Our study was limited by its retrospective nature, relying on the accuracy of notes written at the time of consultation. Secondly, as this is a retrospective study, focus was not on optimizing the Wells scoring or the radiological examinations. The Wells score, and radiological examinations were made by different clinicians and might have had very large interindividual variation. We only used the first US although the recommendations are, that a negative US should be repeated, if D-dimer is positive. However, in the case of more DVT after a second US, our conclusion would have been even stronger. Finally, we did not distinguish between proximal and distal DVT.

\section{Conclusion}

In conclusion, this retrospective study does not support that higher cut-off levels for D-dimer, in the group with low or intermediate Wells scores are safe to exclude the diagnosis of DVT in the elderly admitted to an emergency department.

\section{References}

1. Wells PS, Anderson DR, Bormanis J, Guy F, Mitchell M, et al. (1997) Value of assessment of pretest probability of deep-vein thrombosis in clinical management. Lancet 350:1795-1798. [Crossref]

2. van Es N, Kraaijpoel N, Klok FA, Huisman MV, Den Exter PL, et al. (2017) The original and simplified Wells rules and age-adjusted D-dimer testing to rule out pulmonary embolism: an individual patient data meta-analysis. J Thromb Haemost 15 : 678-684. [Crossref] 
Ramberg E (2018) Will the use of a raised or age-adjusted D-dimer cut-off value in combination with a sequential strategy using the Wells score safely exclude deep venous thrombosis?

3. Hogg K, Wells PS, Gandara E (2012) The diagnosis of venous thromboembolism. Semin Thromb Hemost 38: 691-701. [Crossref]

4. Tripodi A (2011) D-dimer testing in laboratory practice. Clin Chem 57: 1256-1262. [Crossref]

5. Olson JD, Cunningham MT, Higgins RA, Eby CS, Brandt JT (2013) D-dimer: simple test, tough problems. Arch Pathol Lab Med 137: 1030-1038. [Crossref]

6. Mullier F, Vanpee D, Jamart J, Dubuc E, Bailly N, et al. (2014) Comparison of five D-dimer reagents and application of an age-adjusted cut-off for the diagnosis of venous thromboembolism in emergency department. Blood Coagul Fibrinolysis 25: 309-315. [Crossref]

7. Goodacre S, Sampson FC, Sutton AJ, Mason S, Morris F (2005) Variation in the diagnostic performance of D-dimer for suspected deep vein thrombosis. QJM 98: 513 527. [Crossref]

8. Goodacre S, Sutton AJ, Sampson FC (2005) Meta-analysis: The value of clinical assessment in the diagnosis of deep venous thrombosis. Ann Intern Med 143: 129-139. [Crossref]

9. Konstantinides SV (2014) 2014 ESC Guidelines on the diagnosis and management of acute pulmonary embolism. Eur Heart J 35: 3145-3146. [Crossref]

10. Bounameaux H, Perrier A, Righini M (2010) Diagnosis of venous thromboembolism: an update. Vasc Med 15: 399-406. [Crossref]

11. Harper PL, Theakston E, Ahmed J, Ockelford P (2007) D-dimer concentration increases with age reducing the clinical value of the D-dimer assay in the elderly. Intern Med J 37:607-613. [Crossref]

12. Haase C, Joergensen M, Ellervik C, Joergensen MK, Bathum L (2013) Age- and sexdependent reference intervals for D-dimer: evidence for a marked increase by age. Thromb Res 132: 676-680. [Crossref]

13. Puurunen MK, Gona PN, Larson MG, Murabito JM, Magnani JW, et al. (2016) Epidemiology of venous thromboembolism in the Framingham Heart Study. Thromb Res 145: 27-33. [Crossref]

14. Righini M, Nendaz M, Le Gal G, Bounameaux H, Perrier A (2007) Influence of age on the cost-effectiveness of diagnostic strategies for suspected pulmonary embolism. $J$ Thromb Haemost 5: 1869-1877. [Crossref]

15. Haas FJ, Schutgens RE, Biesma DH (2009) An age-adapted approach for the use of D-dimers in the exclusion of deep venous thrombosis. Am J Hematol 84: 488-491. [Crossref]

16. Raviv B, Israelit SH (2012) Shifting up cutoff value of d-dimer in the evaluation of pulmonary embolism: a viable option? Possible risks and benefits. Emerg Med Int 2012: 517375. [Crossref]

17. Douma RA, le Gal G, Söhne M, Righini M, Kamphuisen PW, et al. (2010) Potential of an age adjusted D-dimer cut-off value to improve the exclusion of pulmonary embolism in older patients: a retrospective analysis of three large cohorts. BMJ 340: c1475. [Crossref]
18. Douma RA, Tan M, Schutgens RE, Bates SM, Perrier A, et al. (2012) Using an agedependent D-dimer cut-off value increases the number of older patients in whom deep vein thrombosis can be safely excluded. Haematologica 97:1507-1513. [Crossref]

19. Nybo M, Hvas AM (2017) Age-adjusted D-dimer cut-off in the diagnostic strategy for deep vein thrombosis: a systematic review. Scand J Clin Lab Invest 77: 568-573. [Crossref]

20. Han C, Zhao Y, Cheng W, Yang J, Yuan J, et al. (2015) The performance of age-adjusted D-dimer cut-off in Chinese outpatients with suspected venous thromboembolism. Thromb Res 136:739-743. [Crossref]

21. Wells PS, Anderson DR, Rodger M, Forgie M, Kearon C, et al. (2003) Evaluation of D-dimer in the diagnosis of suspected deep-vein thrombosis. N Engl J Med 349: 1227 1235. [Crossref]

22. Bleker SM, Coppens M, Middeldorp S (2014) Sex, thrombosis and inherited thrombophilia. Blood Rev 28: 123-133. [Crossref]

23. Roach RE, Lijfering WM, Rosendaal FR, Cannegieter SC, le Cessie S (2014) Sex difference in risk of second but not of first venous thrombosis: paradox explained Circulation 129:51-56. [Crossref]

24. Cohen AT, Agnelli G, Anderson FA, Arcelus JI, Bergqvist D, et al. (2007) Venous thromboembolism (VTE) in Europe. The number of VTE events and associated morbidity and mortality. Thromb Haemost 98: 756-764. [Crossref]

25. van Es J, Mos I, Douma R, Erkens P, Durian M, et al. (2012) The combination of four different clinical decision rules and an age-adjusted D-dimer cut-off increases the number of patients in whom acute pulmonary embolism can safely be excluded. Thromb Haemost 2012, 107:167-171. [Crossref]

26. Wilts IT, Le Gal G, Den Exter PL, Van Es J, Carrier M, et al. (2017) Performance of the age-adjusted cut-off for D-dimer in patients with cancer and suspected pulmonary embolism. Thromb Res 152: 49-51. [Crossref]

27. Wilts IT, Le Gal G, den Exter PL, van Es J, Carrier M, et al. (2016) PO-29 - Ageadjusted D-dimer cutoff level increases the number of cancer patients in who pulmonary embolism can be safely excluded without CT-PA imaging: The ADJUST-PE cancer substudy. Thromb Res 140: S187. [Crossref]

28. Gomez-Jabalera E, Bellmunt Montoya S, Fuentes-Camps E, Escudero Rodriguez JR (2018) Age-adjusted D-dimer for the diagnosis of deep vein thrombosis. Phlebology 33: 458-463. [Crossref]

29. Takach Lapner S, Julian JA, Linkins LA, Bates SM, Kearon C (2016) Questioning the use of an age-adjusted D-dimer threshold to exclude venous thromboembolism: analysis of individual patient data from two diagnostic studies. J Thromb Haemost 14: 1953-1959. [Crossref]

30. Wang B, Lin Y, Pan FS, Yao C, Zheng ZY, et al. (2013) Comparison of empirical estimate of clinical pretest probability with the Wells score for diagnosis of deep vein thrombosis. Blood Coagul Fibrinolysis 24: 76-81. [Crossref]

31. Sartori M, Cosmi B, Legnani C, Favaretto E, Valdré L, et al. (2012) The Wells rule and D-dimer for the diagnosis of isolated distal deep vein thrombosis. J Thromb Haemost 10: 2264-2269. [Crossref]

Copyright: (C2018 Ramberg E. This is an open-access article distributed under the terms of the Creative Commons Attribution License, which permits unrestricted use, distribution, and reproduction in any medium, provided the original author and source are credited. 